\title{
Window of Imagination
}

\author{
An Analysis of Graphic Montage Language in Movie Posters
}

\author{
Zhaohui Yuan \\ College of Fine Arts \\ Huanggang Normal University \\ Huanggang, Hubei, China
}

\begin{abstract}
The graphical montage language of the graph in the movie poster opens the window of the fantasy imagination of the movie world for us, and stimulates the enthusiasm of the audience to appreciate the movie with interest. As a graphical montage language of artistic thinking, in the design of the movie poster, the weaving of the episodic narrative is used, which expands the connotation of symbolic significance, presents the connotation of the imagery, instantly conveys the movie information to the audience, creates the suspense, interprets the rich connotation of the movie and shows the spirit and age value of the film.
\end{abstract}

Keywords-movie poster; graphics; montage

\section{INTRODUCTION}

It has been 110 years since the beginning of the film art. Starting from the initial "photography", the film integrates ongoing technological innovation with other art forms, gradually develops into a multi-dimensional comprehensive "modern art". It has become an important cultural entertainment products in modern society, with its abundant subject matters, various forms of art to guide the people's understanding and thinking to the world and change people's life and behavior habit and even mental outlook.

If the film is the imagination world of human creation, the movie poster is the window of looking the imaginary world. As movie derivatives, except the introduction of the film type, plot summary, cast and crew lists and other information to promote sales, with montage psychological effect, movie poster also stimulates the imagination of the film. The meaning of Montage was originally a composition and assembly, originally as the architectural terminology, was referred to use in the motion picture technique. Montage theory in the practice of the film art breaks through unceasingly, gradually forms the perfecting the art of thinking, at the same time, it is also widely used in the design of the poster. In the research and analysis of the movie poster in the history, we can see that the good movie posters on the design fully use the graphical montage language, show the characteristic of the design method, aesthetic thoughts and style schema, accurately grasp the connotation and the theme of the film and show the spirit delivered by the film.

Fund project: advantages superior subject-group construction project funding of Huanggang Normal College.

\section{ARRANGEMENT OF GRAPHICAL MONTAGE EPISODIC NARRATION}

Story is indispensable in the historical evolution of human culture. People use a variety of ways to tell the story of inheriting civilization. Generally, it is considered that language is the main medium of narrative, as a matter of fact, graphic narrative is prior to writing, and the graph is the initial visual form of the human knowing the world, imagining the world, recording the world. The history of graphics can be traced back to prehistoric cave paintings and hieroglyphics. Those graphics in the process of evolution and symbolization were invariably covering the unknown legend stories.

Movie art is telling the story with plot, timeliness and spatiality. The graphic narrative as a type of image narrative, usually its performance is only two-dimensional instantaneous. In movie posters of graphic design, the designers use the montage thought, creatively in two-dimensional space expression, show the flow of the plot. The graphics are no longer to imitate and portray reality. When people admire the montage graphics, the thinking is infinitely extending outward. The main techniques of montage initially was collage, which was a skills used by Picasso and Bouraq when analyzing the Cubist Period. The early photography on narrative imitation, the various collages were used, and in the modern art genre, cubism, Dadaism, modern expressionism, surrealism art view also coincides were happening to have the same view as montage thinking, made the combination of the images of different time and space structure to express the broader concept of space and time. Therefore, "to make such an image, which has become a space time slice to achieve the objective of the narrative, we must make it reflect or imply movement, and we must put it back into the process of the time."

Static, isolated graphic elements do not have the motility of time, but the movie poster design "according to the content creation form, through form convey meaning, in the visual form, constitutes the visualization of the story". Looking for certain logic is by using the montage thought, to constitute the storytelling of visualization. This graphical montage of using collage and combination actually is "emplotment". What movie poster faces is full of the audience who are curious about the story. The plot revealed from the poster graphics is the most direct and effective attracting method. 
The first sci-fi film in the history of the cinema, " A Trip to the Moon", describes the industrial age has brought the rapid development of science and technology; humans began to view the universe. Movie poster graphic depicts the scientists flew into space aboard a shells like spacecraft, and the impact on the moon's left eye, the moon showed surprise, injured facial expressions, but also hinted at in the plot against the aliens on the moon, due to human attacked on the moon, it caused a coherent conflict "Fig. 1". In the time when film art mainly focused on the Lumiere Brothers' documentary movies, the film "A Trip to the moon" was narrating the story of a science fiction adventure story. In the design, the poster was series connecting graphic elements related to the story anthropomorphically, and used the montage way of thinking to make the vision narrating, and with the fantasy narrative pattern, weaved the main plot of the story in the movie, made the audience able to understand the contents of the film to be told.

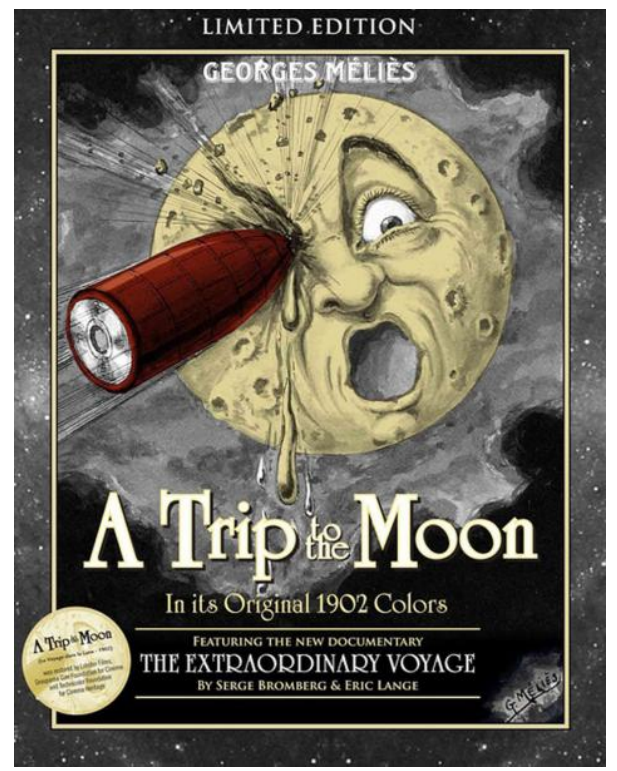

Fig. 1. Poster of the movie "A Trip to the Moon"

\section{Douban Movie}

http://www.douban.com/group/topic/46638187/?author=1

The weaving of the episodic narrative in movie poster design has after that time become a major design mode. Many film poster designs made the combination of collage for information of segments of the plot, scene space, role, still according to the conveying of the film, by using montage language logic. The audience interpreted these events according to the associated graphics, tried to figure out the story content, and movie posters also rely on the words of these incomplete to reveal the suspense of the story, to inspire the watching desire of the audience.

\section{THE EXTENSION OF THE GRAPHICAL MONTAGE SYMBOLIC SIGNIFICANCE}

The original graphics of our ancestors used for communication and thinking is a kind of specific image symbols. The sign and symbol in English is all meaning symbol. The sign has strong concentration and unity, and the symbol and universal concept of things are closer, with broad "sub-consciousness" implied in it. "Symbol uses the image of the external emotional things to suggest an abstract, universal significance. What symbol brings to people or to make people realize it is not this specific and direct significance that the external thing has, but rather is the universal significance implied by certain features"

Jerry N Uelsmann Jerry, American surrealism photographer used the traditional darkroom technology. In his work, he often overlapped and outlined the tree, river, vessels, character and inanimate object and other common elements, broke our habit of thinking "Fig. 2". The turning is from photos to "image narrative" to create a dream-like montage, to bring us wonderful visual enjoyment and psychological experience. The combination of the element significance of those different negatives produces a symbol isomorphism relation, and creates new situational dialogue between elements and expresses the potential consciousness and spirit.

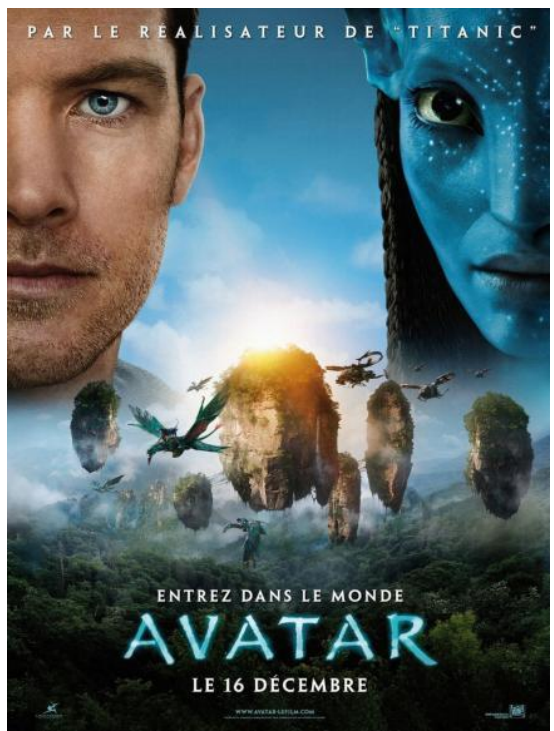

Fig. 2. Poster of the movie "Avatar"

\section{Douban Movie}

http://movie.douban.com/photos/photo/1630382053/

In the poster of the movie "The Silence of the Lambs", the montage collocation technique is used for the graph, and in the features of a corpse facial pale woman, a moth seals the character's mouth to represent the meaning of silence "Fig. 3". Moth in the west has the moral of death metamorphosis, as a kind of symbolic graphic element, there are multiple meanings in the poster. In classic Chinese love story, Liang Shan Bo And Zhu Yingtai turned into a butterfly after the death, were whirling in dancing in the world, so the symbolic meanings should be derived from the moth reproduction characteristics of insects, is a kind of universal meaning implied by the feature. And in the movie "Double-sided Lawrence" , there was a metaphor of butterfly flying out of Lawrence's mouth, and the poster of "The Silence of the Lambs", the audience who look it carefully will find this "hell insect" head is composed of skull. Whether it refers to the 
moth's metamorphosis, or the transition of the human nature, has suggested that the plot development of the story, in the plot of the film, it was the psychological twist desire of amuck, Buffalo Bill that through the skin of woman to give self a beautiful degeneration. There were many times in the bible stories in the film to metaphor the ending. The term "lamb" in Christian culture originally is the synonym of silence, slaughter. In the poster, the surrealism combination of pale woman color skin and bright "hell insects" makes people thriller and unstable.

The film "The Silence of the Lambs" poster graphics is not only simply using the juxtaposition technique of montage, more important is adopting the symbolism between some relevant properties of things, from the psychological effects of montage structures play to build voice of all, makes the poster bringing more thinking to the audience. As a single point graphic element is a single, and the element produced by two isomorphism symbol of montage has multiple significances.

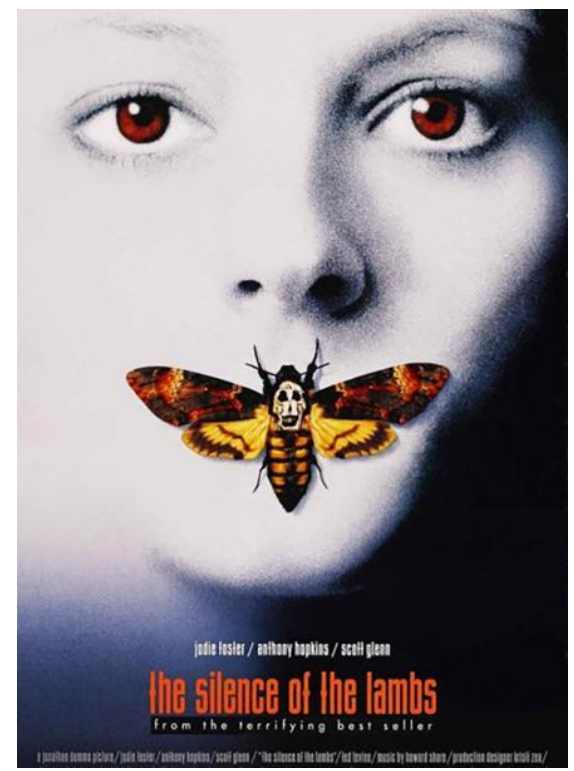

Fig. 3. Poster of the movie "The Silence of the Lambs "

Sea movie - Shanghai film group co., Ltd

http://www.sfs-

cn.com/node3/ypjs/node15741/node15742/u1a1432140.html

\section{The Presentation of Graphical Montage IMAGERY CONNOTATION}

Gestalt theory considers that attention and perception are closely linked, when the visual styles reflected in the external things and graphic form of style are consistent with the human emotional experience; the visual form has the emotional nature. In the vaudeville montage theory, Eisenstein used the strong infection method from composite film, "it is not just any details, but also has 'overall feeling feature and the details of the element. Caused by the own "part", Juggling montage strong appeals overall influence on the audience's emotions with part. We can understand that this montage is a kind of image performance. It is not reality, but reveals the reality of the world, is a kind of integration of subjective aesthetic emotion and objective image artistic mean.

Juggling montage was criticized too formalism, but, as in the painting, the relation between the whole and local, and the image shows of montage is a partial gathering based on complete meaning, and converts the connotation into the spiritual outlook of the expression transfer graphics visually.

Chinese traditional culture emphasizes a kind of "theory that man is an integral part of nature" Chinese traditional culture ideal state, lays emphasis on the affinity between man and nature, to achieve the integration of the perceptual image with their own state of mind of "image", and becomes the essence of Chinese traditional aesthetic thought. In graphical montage language, it is expressed through the elements of the perceptual visual form. Watching is a kind of fuzzy representation, and expresses the subjective aesthetic imagery and spiritual pursuit. In the poster of the domestic industry "Golden Age" of series, the dark splash-ink, blade character of the jungle, a symbol of the troubled times, the heroine of loneliness, stubbornly stands in the depressive space "Fig. 4". The text and ink is the power source of the heroine as a writer. The small potato is caught in the flood current of the great age. The strong psychological impact caused by the comparison of stillness and activeness strong and weak visually, in the imagery of the graphical montage of reality with the real transformation, shows a contradictory emotion and hot pursuit of spirit. In the spirit transmission of the building of graphical montage, the spirit "grasping the information of the image of character, converts its connotation into a graphic visual expression of interest, through the integration of inner implication and external form, to show its appeal" and the graphics image infection makes the graphics reach the resonate with the audience, so that transfers the spirit of the film to the audience and to reach the final theme of the film.

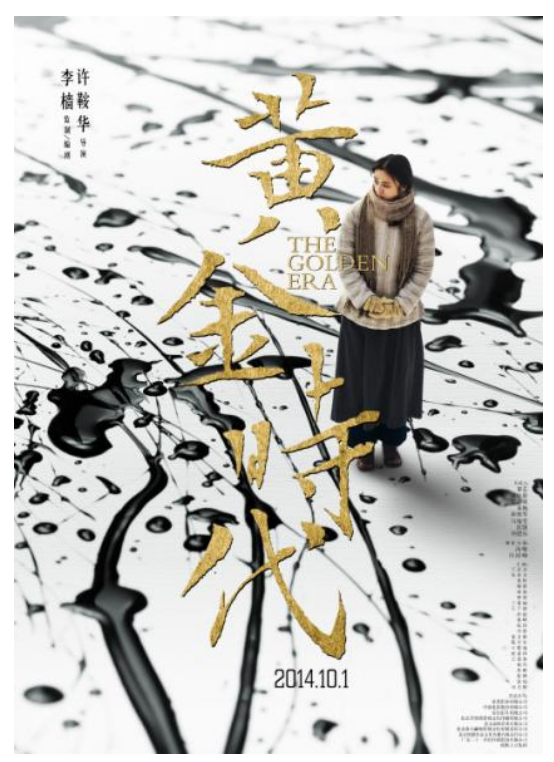

Fig. 4. Poster of the movie "Golden Age"

Sea movie - Shanghai film group co., Ltd

http://www.sfs-

cn.com/node3/node625/node3034/userobject1ai1530948.html 
The imagery of Graphical montage is the graphic expression of the creatively to the spiritual world. With the image infection of the overall to cause the emotional resonance, to make the audience first time able to enter the story situation, and is closer to express the spirit that the movie ontology wants. The imagery graphical language makes the style of the film poster design permeate a unique aesthetic value.

\section{CONCLUSION}

The graphical montage language of the movie posters opens the first window of film for us, shows the movie world fantasy imagination, arouses the enthusiasm of the audience to appreciate the movie with interest and promotes the development of film art. The cultural and economic development and social progress has higher requirements to the movie and movie poster design. The graphic design in the movie posters needs extensive reference and use of other artistic thinking and expression form to become an era of social and cultural microcosm, continually interprets the colorful cinematographic work.

\section{REFERENCES}

[1] Long Diyong: Graphical Narrative: Temporizing of space [J], Jiangxi Social Sciences, 2007.9, p.42.

[2] Yuan Zhaohui: Graphical montage language [J], Art Panorama, 2009, p.228.

[3] Liu Xicheng: Symbol - the investigation on a folk cultural patterns [M], academe publishing house, 2002, page 8 .

[4] (Russian) $\mathrm{C} \cdot \mathrm{M} \cdot$ Eisenstein: Montage Theory [M], China Film Press, 2003, p. 185.

[5] Cao Fang: Visual Communication Principles, Jiangsu Fine Arts Publishing House [M], 2005, p. 276. 